\title{
Establishing cellular stress response profiles as biomarkers of homeodynamics, health and hormesis
}

\author{
Dino Demirovic*, Suresh I.S. Rattan \\ Laboratory of Cellular Ageing, Department of Molecular Biology and Genetics, Aarhus University, Aarhus, Denmark
}

\section{A R T I C L E I N F O}

Article history:

Received 6 December 2011

Received in revised form 26 January 2012

Accepted 7 February 2012

Available online 22 February 2012

Section Editor: A. Simm

\section{Keywords:}

Autophagy

Health

Hormesis

Hormetins

Heat shock

Nutrition

Stress

\begin{abstract}
A B S T R A C T
Aging is the progressive shrinkage of the homeodynamic space. A crucial component of the homeodynamic space is the stress response (SR), by virtue of which a living system senses disturbance and initiates a series of events for maintenance, repair, adaptation, remodeling and survival. Here we discuss the main intracellular SR pathways in human cells, and argue for the need to define and establish the immediate and delayed stress response profiles (SRP) during aging. Such SRP are required to be established at several age-points, which can be the molecular biomarkers of homeodynamic space and the health status of cells and organisms. SRP can also be useful for testing potential protectors and stimulators of homeodynamics, and can be a standard for monitoring the efficacy of potential pro-survival, health-promoting and aging-modulating conditions, food components and other compounds. An effective strategy, which makes use of SRP for achieving healthy aging and extending the healthspan, is that of strengthening the homeodynamics through repeated mild stress-induced hormesis by physical, biological and nutritional hormetins. Furthermore, SRP can also be the basis for defining health as a state of having adequate physical and mental independence of activities of daily living, by identifying a set of measurable parameters at the most fundamental level of biological organization.
\end{abstract}

(c) 2012 Elsevier Inc. All rights reserved.

\section{Introduction}

A fundamental characteristic of all living system is the ability to respond, counteract, and adapt to internal and external sources of disturbance. This ability is traditionally termed as homeostasis, which is a somewhat misleading term since there is little or no constancy in living systems. Rather it is the dynamic equilibrium of continuous changes and remodeling that determines survival, and for which the term homeodynamics (Yates, 1994) is better suited to expound the continuity of growth, development and aging. The term homeodynamics has been further modified to homeodynamic space as indicative of the overall survival ability and the buffering capacity of a biological system (Holliday and Rattan, 2010; Rattan, 2006, 2007, 2011).

One of the crucial components of the homeodynamic space is the stress response (SR), by virtue of which a living system is able to sense disturbance, and initiates a series of events for maintenance, repair, adaptation, remodeling and survival. There are three main aspects of SR: (1) immediate SR involving extra- and intracellular signaling during the period of disturbance and exposure to stressors; (2) delayed SR involving sensors and modulators in the presence of stressors or after the removal of stressors; and (3) downstream

\footnotetext{
* Corresponding author.

E-mail address: did@mb.au.dk (D. Demirovic).
}

effectors for counteracting the effects of disturbance and for reestablishing homeodynamics. At present it is not known how these three steps are maintained interactively in terms of kinetics and intensity, and how these may alter during growth, development and aging. In order to develop novel and effective means of aging modulators and maintainers of homeodynamics, it is crucial to elucidate the nature and effects of immediate and delayed SR in cells and tissues undergoing aging.

In this article, we briefly review the main intracellular SR pathways in human cells, and discuss the urgency of defining and establishing the immediate and delayed stress response profiles (SRP) of normal cells. Such SRP are required to be established at several agepoints, and can be used as molecular biomarkers of homeodynamic space and the health status of cells and organisms. SRP can be very useful for testing potential protectors and stimulators of homeodynamics, and can be a kind of "gold-standard" for monitoring the efficacy of potential pro-survival, health-promoting and aging-modulating conditions and compounds.

\section{Stress responses $(\mathrm{SR})$}

The term SR is defined as a response by cells, tissues and organisms to any physical, chemical or biological factor(s), which initiates a series of biological events that facilitate and promote counteraction, adaptation and survival. At the intracellular and molecular level, mammalian cellular SR can be categorized into seven main distinct 
pathways which are integral to the organismic property of homeodynamics (Table 1). Based on the involvement of one or more molecular SR, higher order (cellular, organ level and body level) SR is manifested, which include apoptosis, inflammation, thermoregulation and hyper-adrenocorticism (Rattan and Demirovic, 2010a). Not all pathways of SR respond to every stressor, and although there may be some overlap, generally SR pathways are quite specific.

The specificity of SR is mostly determined by the nature of the disturbance or the damage induced by the stressor and the variety of downstream effectors involved. For example, cytoplasmic induction of protein denaturation initiates the so-called heat shock response (HSR) by activating heat shock transcription factors (HSF), and by inducing the transcription and translation of several heat shock protein (HSP) genes (Liberek et al., 2008; Verbeke et al., 2001). However, unfolded proteins in the endoplasmic reticulum (ER) induce the unfolded protein response (UPR), and initiate the induction of synthesis of a totally different set of proteins and their downstream effectors, such as GRP78/BiP, GRP94/gP96, GRP170/ORP150, GRP58/ERp57, PDI, Erp72, calnexin, calretuculin , EDEM, Herp and other co-chaperones (Banhegyi et al., 2007; Lin et al., 2007; Ni and Lee, 2007; Yoshida, 2007). Similarly, a mitochondria-specific SR involves the induction and activation of various other chaperones, such as chaperonin-10 (Cpn10/Hsp10), chaperonin-60 (Cpn60/Hsp60), and mortalin (Kaul et al., 2007; Zhao et al., 2002).

Another well-known SR is the oxidative stress response, in which cells respond to oxidative stressors through the regulation of transcription of several antioxidant genes. The main regulator of this specific antioxidant phenotype is the nuclear factor-erythroid-2 (Nrf2) transcription factor, which regulates the basal and inducible expression of numerous detoxifying and antioxidant genes (Ishii et al., 2002). Under normal conditions Nrf2 is held in the cytoplasm by the specific inhibitory protein KEAP1. Oxidative modification of cysteine residues of KEAP1 induces conformational changes and a loss of Nrf-2 binding, allowing Nrf2 to translocate to the nucleus where it heterodimerizes with specific co-factors, and leads to the transcription of various genes through the regulatory regions of antioxidant response elements (Motohashi and Yamamoto, 2004; Yamamoto et al., 2004). Some of the genes activated by stress-induced activation of Nrf2 are heme-oxygenase1 (HO-1), NAD(P)H-quinone oxidoreductase-1 (NQO1), and GST mRNA and proteins in rodent tissues (Calabrese, 2008; Pearson et al., 2008).

Activation of DNA repair enzymes in response to DNA damage is a SR which is essential for the maintenance of genomic stability (Hakem, 2008; Vijg and Campisi, 2008). DNA damage response (DDR) involves a variety of transcription factors being translocated to the nucleus, binding the double- and single-strand DNA breaks, and activating the transcription of several DNA repair enzymes. Other SR involve activation of sirtuins causing deacetylation of histones and other proteins in response to reduced levels of metabolic energy (Longo, 2009; North and Sinclair, 2007); activation of NFkBmediated cytokine synthesis (Yeung et al., 2004); and the autophagy response which is the lysosomal-mediated and chaperone-mediated sequestering of damaged membranes and organelles induced during nutritional limitation, starvation, and hypoxia (Markaki and Tavernarakis, 2011; Martinez-Vicente et al., 2005; Terman et al., 2007; Yen and Klionsky, 2008).

However, an induction of a specific SR pathway as the first response (immediate response) does not rule out the induction of one or more other SR pathways later on (delayed response). A complete and successful SR for effective homeodynamics and for the maintenance of the homeodynamic space includes both immediate and delayed SR. It is therefore important that all SR pathways are analysed simultaneously and a complete SRP is established under a given condition, such as age-, health- and disease status, and during and after exposure to single or multiple stressors.

\section{Stress response profiles (SRP)}

Determining SRP is essential for establishing the nature and extent of the homeodynamic space of cells, tissues and organisms. Furthermore, being able to map the kinetics and amplitude of different SR, and their effects on each other, can form the basis to evaluate the health status of an individual and to develop effective means of aging modulators and maintainers of homeodynamic space.

In Fig. 1, two theoretical SRP are visualized for an idealized healthy young cell exposed to either heat shock (HS) or nutritional deprivation for a short period. Fig. 1A represents the virtual SRP in response to HS, in which HSR is the primary response and is set to reach the maximum in a given period of time. There is a large body of published data for HSR in various biological systems (Richter et al., 2010; Verbeke et al., 2001), but little is known with respect to the other SR pathways during and after HS. For a complete SRP, one needs to study the other six pathways in the same samples during the same duration and beyond. Obviously, not all pathways will respond equally in time and amplitude, and even some pathways may be suppressed due to the limitation of energy resources. For example, it has been proposed that autophagy may be triggered as a late response after exposure to HS (Zhao et al., 2009). Similarly, whereas one can expect to see UPR, inflammatory response and sirtuin response to varying extents as delayed responses to HS, it is unlikely that any significant DDR may occur during or after HS (Richter et al., 2010).

Another example of our theoretical SRP (Fig. 1B), envisages the behavior of the stress pathways after nutritional deprivation, in which case the autophagic response is expected to be the primary response (Rabinowitz and White, 2010). Autophagy is a conserved response initiated by the inhibition of the mammalian target of rapamycin (mTOR) complex, resulting in the formation of a membrane-encapsulated

Table 1

Main molecular level stress response pathways and their respective inducers and effectors in human cells. Modified from Rattan and Demirovic (2010b).

\begin{tabular}{|c|c|c|c|}
\hline Response & Stressors & Effectors & Molecular markers \\
\hline Heat shock response (HSR) & $\begin{array}{l}\text { Heat, heavy metals, antibiotics, protein } \\
\text { denaturation }\end{array}$ & $\begin{array}{l}\text { Heat shock proteins, proteasome and } \\
\text { other proteases }\end{array}$ & $\begin{array}{l}\text { Translocation of HSF-1 into the nucleus; induction of HSP72 } \\
\text { (HSPA1A) }\end{array}$ \\
\hline Unfolded protein response (UPR) & Unfolded and misfolded proteins in ER & Chaperones and co-chaperones & Activation of transcription factor 6 (ATF-6); CHOP (GADD153) \\
\hline Autophagic response & $\begin{array}{l}\text { Food limitation, hypoxia, damaged } \\
\text { organelles }\end{array}$ & Autophagosomes, Lysosomes & $\begin{array}{l}\text { Altered LC3-I and LC3-II ratio; increased number of lysosomes; } \\
\text { degradation of damaged mitochondria }\end{array}$ \\
\hline DNA damage response (DDR) & Radiation, oxidants, free radicals & DNA repair enzymes & $\begin{array}{l}\text { Translocation of ATRIP to the nucleus; increased levels of } \\
\text { checkpoint proteins p53, p16, p21, mortalin }\end{array}$ \\
\hline Antioxidant response & $\begin{array}{l}\text { Free radicals, reactive oxygen species, } \\
\text { pro-oxidants }\end{array}$ & Nrf-2, heme oxygenase, FOXO & HO-1; FOXO protein levels \\
\hline Sirtuin response & Energy depletion & Sirtuins & Sirtuin-1 protein level; NAD/NADH ratio \\
\hline Inflammatory response & $\begin{array}{l}\text { Pathogens, allergens, damaged } \\
\text { macromolecules }\end{array}$ & Cytokines, nitric oxide synthase, COX-2 & Increased level of NF- $\kappa \mathrm{B}$ protein and inflammatory interleukins \\
\hline
\end{tabular}



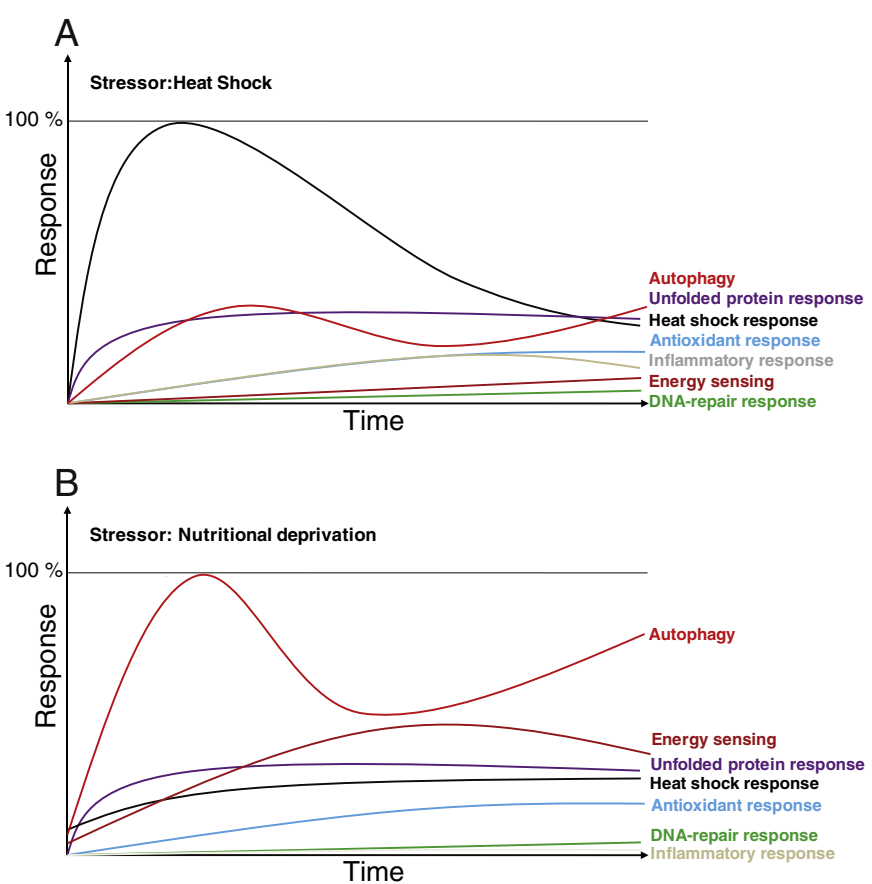

Fig. 1. Virtual stress response profiles (SRP) comprising seven pathways in a healthy young cell. A stressor initially elicits a specific primary stress response, for example heat shock response by heat stress (A), and autophagic response by nutritional deprivation (B), followed by other responses, which may differ widely in terms of kinetics and amplitude.

vesicle, which eventually fuses with lysosomes and undergoes degradation (Markaki and Tavernarakis, 2011). The mature vesicle, autophagosome, usually contains damaged and inactive organelles, misfoldedand aggregated-proteins, and other cytoplasmic components, which are not removed by the proteasomal degradation processes. During nutritional deprivation other stress pathways that are expected to be affected are the energy sensing pathway due to altered NAD/NADH ratio and increasing levels of sirtuins (Cantó et al., 2010). Similarly, there is some indication that activation of autophagy often goes together with ER stress (Ravikumar et al., 2010).

The virtual SRP for HS and nutritional deprivation in Fig. 1 are examples of the interactive and co-dependency of SR pathways. Our experimental studies are now in progress to establish the factual SRP for normal human cells undergoing serial passaging and replicative senescence, using mild and severe HS, various extents of serum deprivation or by inducing nutritional stress-like conditions by rapamycin and caloric-restriction mimetics. Ideally, such SRP should be created for every SR pathway using a primary stressor specific for the pathway, followed by the immediate and delayed response elucidation of all other pathways.

As regards methodoligcal approaches, as an example, we expose normal human skin fibroblasts at different ages during their replicative lifespan in vitro (measured in terms of cumulative population doublings during serial passaging) to either mild $\left(41^{\circ} \mathrm{C}\right)$ or severe $\left(42.5^{\circ} \mathrm{C}\right)$ heat stress for $1 \mathrm{~h}$, or to nutritional stress by reducing serum concentration (from $10 \%$ to $2 \%$ or $0 \%$ ) in the medium for $24 \mathrm{~h}$. At various times, cells are collected and prepared for protein analysis, using standard protocols for ELISA and Western blotting methods, and using appropriate antibodies in accordance with the list of molecular markers given in Table 1 . Since several of the early markers of SR are the transcription factors which mainly become translocated from the cytoplasm to the nucleus as the immediate SR, it is also important to document their translocation by immunofluorescence-microscopy. For quantitative estimation and cell population distribution, antibodies-associated fluorescence can also be analysed using a flow cytometer. A combination of ELISA and Western blotting, together with a visual documentation of transcription factor activation by immunofluorescencemicroscopy, can give sufficient information to generate the immediate SRP. However, at this stage, it is not essential to analyse the global gene expression by measuring the levels of hundreds or thousands of mRNAs using expensive gene array-based technologies, which may be useful for detecting late or delayed SR.

Basic knowledge of the SRP under normal healthy conditions, and at different age-points during the limited lifetime of cells and organisms, needs to be acquired as a reference. In a search for biomarkers of aging, much attention has been paid to single molecules, factors, or proteins which will either be up- or down-regulated with increasing age (Malavolta et al., 2010). However, in order to address the issues of health, survival ability and longevity, it is important to elucidate various components of the homeodynamic space in a collective and holistic manner. Since the ability to respond to stress is a crucial component of the homeodynamic space, we suggest that the SRP can be a better biomarker of health and aging.

\section{Stress, hormesis and hormetins}

The consequences of stress can be both harmful and beneficial depending on the intensity, duration and frequency of the stress, and on the price paid in terms of energy utilisation and other metabolic disturbances. But the most important aspect of SR is that it is not monotonic with respect to the dose of the stressor. Rather it is almost always characterised by a nonlinear biphasic relationship. Several metaanalyses performed on a large number of papers published in the fields of toxicology, pharmacology, medicine, and radiation biology have led to the conclusion that the most fundamental shape of the dose response is neither threshold nor linear, but is U- or inverted U-shaped, depending on the endpoint being measured (Calabrese, 2007; Calabrese and Baldwin, 2001). This phenomenon of biphasic dose response is termed as hormesis (Southam and Ehrlich, 1943). The key conceptual features of hormesis are the disruption of homeodynamics, the modest overcompensation, and the reestablishment of homeodynamics.

Hormesis in aging is characterised by the life-supporting beneficial effects resulting from the cellular responses to single or multiple rounds of mild stress (Rattan, 2008a). This is figuratively represented in Fig. 2 that whereas the homeodynamic ability of a biological system is strengthened in a hormetic zone $(\mathrm{H})$ during mild stress, chronic and severe stress results in the progressive weakening of homeodynamics and

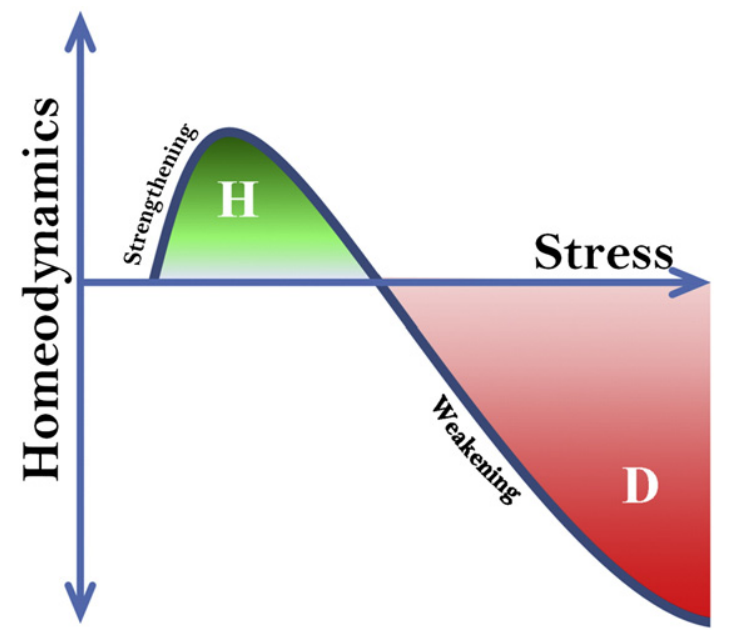

Fig. 2. The homeodynamic ability of a biological system is affected by stress in a biphasic dose response manner, termed physiological hormesis. Lower levels of stress result in the strengthening of homeodynamics in a hormetic zone $(\mathrm{H})$, and chronic and severe stress result in the weakening and disruption (D) of the homeodynamics leading to functional impairments, diseases and eventual death. 
an increased zone of disruption (D) leading to functional impairments, diseases and eventual death. It is important to note that whereas the hormetic zone is usually small both with respect to the dose and the effect, its biological consequences can be cumulative, amplified and physiologically significant. All such conditions, which bring about biologically beneficial effects by causing mild stress, by activating one or more SR pathways and by strengthening the homeodynamics, are termed as hormetins (Rattan, 2008a; Rattan and Demirovic, 2010b; Rattan et al., 2009). Hormetins may be further categorized as: (1) physical hormetins, such as exercise, heat and radiation; (2) psychological hormetins, such as mental challenge and focused attention or meditation; and (3) biological and nutritional hormetins, such as infections, micronutrients, spices and other sources (Rattan, 2011).

An example of stress-induced hormesis is the well-documented beneficial effects of moderate exercise as a hormetin, which initially increases the production of free radicals, acids and aldehydes (Landi et al., 2010; Radak et al., 2008). Another frequent observation in studies of hormesis is that a single hormetic agent, such as HS or physical activity, can strengthen the overall homeodynamics of cells and enhance other activities such as tolerance to other stresses, by initiating a cascade of processes resulting in a biological amplification and eventual beneficial effects (Mattson, 2008; Rattan, 2008a).

Various mild stresses that have been reported to delay aging and prolong longevity in cells and animals include temperature shock, irradiation, heavy metals, pro-oxidants, acetaldehyde, alcohols, hypergravity, exercise and food restriction (Le Bourg and Rattan, 2008). Aging modulatory and other effects of hormesis have been reported for human cells. For example, using a regimen of repeated mild heat shock (RMHS) given to cultured normal human skin fibroblasts, keratinocytes, endothelial cells, and telomerase-immortalised bone marrow mesenchymal stem cells, we have reported a variety of hormetic effects. These effects include slowing down of cellular aging, some extension of replicative lifespan, maintenance of youthful morphology, reduction in the levels of oxidatively- and glycoxidatively-damaged proteins, stimulation of proteasomal activities, increased levels of chaperones, enhancement of stress tolerance, and improvement in differentiation, wound healing and angiogenesis (Rattan et al., 2009). Other hormetic conditions, which have been shown to have anti-aging effects in human cells are irradiation, mechanical stretching, and electromagnetic field shock (Demirovic and Rattan, 2011; Lima et al., 2011; Moskalev et al., 2011; Perez et al., 2008; Rattan, 2008b).

Nutritional hormetins, especially those derived from plant sources, have generated much scientific interest for their health beneficial effects. This is because of the realization that not all chemicals found in plants are beneficial for animals in a simple and straightforward manner, but rather they cause molecular damage by virtue of their electrochemical properties and have a typical biphasic hormetic dose response (Balstad et al., 2011). Although the exact nature of the initial molecular damage caused by such compounds may not be easily identified, an activation of one or more SR (Table 1) is a good indicator of the primary action of the compound. Some examples of nutritional hormetins involving HSR are phenolic acids, polyphenols, flavanoids, ferulic acid (Barone et al., 2009; Son et al., 2008), geranylgeranyl, rosmarinic acid, kinetin, zinc (Berge et al., 2008; Son et al., 2008; Sonneborn, 2010), and the extracts of tea, dark chocolate, saffron and spinach (Wieten et al., 2010).

\section{Conclusions}

In this perspective article, we have argued that optimal SR is a crucial component of the homeodynamic ability of living systems, by virtue of which they initiate a series of events for maintenance, repair, adaptation and remodeling, which are essential for survival and for maintaining health. At the intracellular level, an induction of a specific SR pathway among the seven main pathways (Table 1) does not rule out the induction or suppression of other SR pathways later on. Furthermore, a complete and successful SR for effective homeodynamics and for the maintenance of the homeodynamic space includes both immediate and delayed SR. Therefore, it is important that all SR pathways are analysed simultaneously and a complete profile is established under a given condition, such as age-, health- and disease status, and during and after exposure to mild or severe stress, and single or multiple stressors. Such SRP can be the molecular and functional biomarker of homeodynamic space and help to define the health status of cells and organisms (Fig. 1).

SRP can be of great value in establishing the biological criteria for defining health, which is so far not a well-defined concept. Often "health" is described either in the context of the absence of one or more diseases or as a vague concept of well-being, without having any objective measures for that. Although some parameters of frailty have been proposed (Fulop et al., 2010; Hubbard and Woodhouse, 2010; Montesanto et al., 2010), measures of health largely remain undefined. Ideally, health can be defined as a state of complete physical and mental independence in activities of daily living, and being healthy implies having adequate physical and mental activities of daily living. However, this definition of health still requires identifying a set of measurable parameters at the most fundamental level of biological organization. Considering SRP as being one of the crucial components of the homeodynamic space provides a possibility to quantify health at the level of cells, tissues and the body. Most importantly, SRP can be useful for testing potential protectors and stimulators of homeodynamics for monitoring the efficacy of pro-survival, health-promoting and aging-modulating conditions, food components and natural and synthetic compounds.

\section{Acknowledgement}

Laboratory of Cellular Ageing is financially partially supported by a research grant from LVMH Recherche, Saint Jean de Braye, France.

\section{References}

Balstad, T.R., Carlsen, H., Myhrstad, M.C., Kolberg, M., Reiersen, H., Gilen, L., Ebihara, K., Paur, I., Blomhoff, R., 2011. Coffee, broccoli and spices are strong inducers of electrophile response element-dependent transcription in vitro and in vivo - studies in electrophile response element transgenic mice. Mol. Nutr. Food Res. 55, 185-197.

Banhegyi, G., Baumeister, P., Benedetti, A., Dong, D., Fu, Y., Lee, A.S., Li, J., Mao, C., Margittai, E., Ni, M., Paschen, W., Piccirella, S., Sensei, S., Sitia, R., Wang, M., Yang, W., 2007. Endoplasmic reticulum stress. Ann. N. Y. Acad. Sci. 1113, 58-71.

Barone, E., Calabrese, V., Mancuso, C., 2009. Ferulic acid and its therapeutic potential as a hormetin for age-related diseases. Biogerontology 10, 97-108.

Berge, U., Kristensen, P., Rattan, S.I.S., 2008. Hormetic modulation of differentiation of normal human epidermal keratinocytes undergoing replicative senescence in vitro. Exp. Gerontol. 43, 658-662.

Calabrese, E., 2007. Threshold-dose-response model - RIP: 1911 to 2006. Bioessays 29, 686-688.

Calabrese, E.J., 2008. Hormesis and medicine. Br. J. Clin. Pharmacol. 66, 594-617.

Calabrese, E.J., Baldwin, L.A., 2001. Hormesis: U-shaped dose responses and their centrality in toxicology. Trends Pharmacol. Sci. 22, 285-291.

Cantó, C., Jiang, L.Q., Deshmukh, A.S., Mataki, C., Coste, A., Lagouge, M., Zierath, J.R., Auwerx, J., 2010. Interdependence of AMPK and SIRT1 for metabolic adaptation to fasting and exercise in skeletal muscle. Cell Metab. 11, 213-219.

Demirovic, D., Rattan, S., 2011. Curcumin induces stress response and hormetically modulates wound healing ability of human skin fibroblasts undergoing ageing in vitro. Biogerontology 12, 437-444.

Fulop, T., Larbi, A., Witkowski, J.M., McElhaney, J., Loeb, M., Mitnitski, A., Pawelec, G., 2010. Aging, frailty and age-related diseases. Biogerontology 11, 547-563.

Hakem, R., 2008. DNA-damage repair; the good, the bad, and the ugly. EMBO J. 27, 589-605.

Holliday, R., Rattan, S.I., 2010. Longevity mutants do not establish any "new science" of ageing. Biogerontology 11, 507-511.

Hubbard, R.E., Woodhouse, K.W., 2010. Frailty, inflammation and the elderly. Biogerontology 11, 635-641.

Ishii, T., Itoh, K., Yamamoto, M., 2002. Roles of Nrf2 in activation of antioxidant enzyme genes via antioxidant responsive elements. Methods Enzymol. 348, 182-190.

Kaul, S.C., Deocaris, C.C., Wadhwa, R., 2007. Three faces of mortalin: a housekeeper, guardian and killer. Exp. Gerontol. 42, 263-274. 
Landi, F., Abbatecola, A.M., Provinciali, M., Corsonello, A., Bustacchini, S., Manigrasso, L. Cherubini, A., Bernabei, R., Lattanzio, F., 2010. Moving against frailty: does physical activity matter? Biogerontology 11, 537-545.

Le Bourg, E., Rattan, S.I.S. (Eds.), 2008. Mild Stress and Healthy Aging: Applying Hormesis in Aging Research and Interventions. Springer, Dordrecht, The Netherlands.

Liberek, K., Lewandowska, A., Zietkiewicz, S., 2008. Chaperones in control of protein disaggregation. EMBO J. 27, 328-335.

Lima, C.F., Pereira-Wilson, C., Rattan, S.I., 2011. Curcumin induces heme oxygenase-1 in normal human skin fibroblasts through redox signaling: relevance for anti-aging intervention. Mol. Nutr. Food Res. 55, 430-442.

Lin, J.H., Li, H., Yasumura, D., Cohen, H.R., Zhang, C., Panning, B., Shokat, K.M., LaVail, M.M., Walter, P., 2007. IRE1 signaling affects cell fate during the unfolded protein response. Science 318, 944-949.

Longo, V.D., 2009. Linking sirtuins, IGF-I signaling, and starvation. Exp. Gerontol. 44, $70-74$.

Malavolta, M., Giacconi, R., Piacenza, F., Santarelli, L., Cipriano, C., Costarelli, L., Tesei, S., Pierpaoli, S., Basso, A., Galeazzi, R., Lattanzio, F., Mocchegiani, E., 2010. Plasma copper/ zinc ratio: an inflammatory/nutritional biomarker as predictor of all-cause mortality in elderly population. Biogerontology 11, 309-319.

Markaki, M., Tavernarakis, N., 2011. The role of autophagy in genetic pathways influencing ageing. Biogerontology 12, 377-386.

Martinez-Vicente, M., Sovak, G., Cuervo, A.M., 2005. Protein degradation and aging. Exp. Gerontol. 40, 622-633.

Mattson, M.P., 2008. Hormesis defined. Ageing Res. Rev. 7, 1-7.

Montesanto, A., Lagani, V., Martino, C., Dato, S., De Rango, F., Berardelli, M., Corsonello, A., Mazzei, B., Mari, V., Lattanzio, F., Conforti, D., Passarino, G., 2010. A novel, population-specific approach to define frailty. Age 32, 385-395.

Moskalev, A., Plyusnina, E., Shaposhnikov, M., 2011. Radiation hormesis and radioadaptive response in Drosophila melanogaster flies with different genetic backgrounds: the role of cellular stress-resistance mechanisms. Biogerontology 12, 253-263.

Motohashi, H., Yamamoto, M., 2004. Nrf2-Keap1 defines a physiologically important stress response mechanism. Trends Mol. Med. 10, 549-557.

$\mathrm{Ni}$, M., Lee, A.S., 2007. ER chaperones in mammalian development and human diseases. FEBS Lett. 581, 3641-3651.

North, B.J., Sinclair, D.A., 2007. Sirtuins: a conserved key unlocking AceCS activity. Trends Biochem. Sci. 32, 1-4.

Pearson, K.J., Lewis, K.N., Price, N.L., Chang, J.W., Perez, E., Cascajo, M.V., Tamashiro, K.L., Poosala, S., Csiszar, A., Ungvari, Z., Kensler, T.W., Yamamoto, M., Egan, J.M., Longo, D.L., Ingram, D.K., Navas, P., de Cabo, R., 2008. Nrf2 mediates cancer protection but not prolongevity induced by caloric restriction. Proc. Natl. Acad. Sci. U. S. A. $105,2325-2330$

Perez, F.P., Zhou, X., Morisaki, J., Jurivich, D., 2008. Electromagnetic field therapy delays cellular senescence and death by enhancement of the heat shock response. Exp. Gerontol. 43, 307-316.

Rabinowitz, J.D., White, E., 2010. Autophagy and metabolism. Science 330, 1344-1348.

Radak, Z., Chung, H.Y., Koltai, E., Taylor, A.W., Goto, S., 2008. Exercise, oxidative stress and hormesis. Ageing Res. Rev. 7, 34-42.

Rattan, S.I.S., 2006. Theories of biological aging: genes, proteins and free radicals. Free Radic. Res. 40, 1230-1238.

Rattan, S.I.S., 2007. The science of healthy aging: genes, milieu, and chance. Ann. N. Y. Acad. Sci. 1114, 1-10.

Rattan, S.I.S., 2008a. Hormesis in aging. Ageing Res. Rev. 7, 63-78.
Rattan, S.I.S., 2008b. Hormetic modulation of aging in human cells. In: Le Bourg, E Rattan, S.I.S. (Eds.), Mild Stress and Healthy Aging: Applying Hormesis in Aging Research and Interventions. Springer, Dordrecht, The Netherlands.

Rattan, S.I.S., 2011. Biogerontology: from here to where? The Lord Cohen Medal Lecture-2011. Biogerontology. doi:10.1007/s10522-011-9354-3.

Rattan, S.I.S., Demirovic, D., 2010a. Hormesis as a mechanism for the anti-aging effects of calorie restriction. In: Everitte, A.V., Rattan, S.I.S., Le Couteur, D.G., de Cabo, R. (Eds.), Calorie Restriction, Aging and Longevity. Springer, Dordrecht.

Rattan, S.I.S., Demirovic, D., 2010b. Hormesis can and does work in humans. DoseResponse 8, 58-63.

Rattan, S.I.S., Fernandes, R.A., Demirovic, D., Dymek, B., Lima, C.F., 2009. Heat stress and hormetin-induced hormesis in human cells: effects on aging, wound healing, angiogenesis and differentiation. Dose-Response 7, 93-103.

Ravikumar, B., Sarkar, S., Davies, J.E., Futter, M., Garcia-Arencibia, M., Green-Thompson, Z.W., Jimenez-Sanchez, M., Korolchuk, V.I., Lichtenberg, M., Luo, S., Massey, D.C.O., Menzies, F.M., Moreau, K., Narayanan, U., Renna, M., Siddiqi, F.H., Underwood, B.R., Winslow And, A.R., Rubinsztein, D.C., 2010. Regulation of mammalian autophagy in physiology and pathophysiology. Physiol. Rev. 90, 1383-1435.

Richter, K., Haslbeck, M., Buchner, J., 2010. The heat shock response: life on the verge of death. Mol. Cell 40, 253-266.

Son, T.G., Camandola, S., Mattson, M.P., 2008. Hormetic dietary phytochemicals. Neuromolecular Med. 10, 236-246.

Sonneborn, J.S., 2010. Mimetics of hormetic agents: stress-resistance triggers. DoseResponse 8, 97-121.

Southam, C.M., Ehrlich, J., 1943. Effects of extracts of western red-cedar heartwood on certain wood-decaying fungi in culture. Phytopathology 33, 517-524.

Terman, A., Gustafsson, B., Brunk, U.T., 2007. Autophagy, organelles and ageing. J. Pathol. $211,134-143$.

Verbeke, P., Fonager, J., Clark, B.F.C., Rattan, S.I.S., 2001. Heat shock response and ageing: mechanisms and applications. Cell Biol. Int. 25, 845-857.

Vijg, J., Campisi, J., 2008. Puzzles, promises and a cure for ageing. Nature 454 1065-1071.

Wieten, L., van der Zee, R., Goedemans, R., Sijtsma, J., Serafini, M., Lubsen, N.H., van Eden, W., Broere, F., 2010. Hsp70 expression and induction as a readout for detection of immune modulatory components in food. Cell Stress Chaperones 15, 25-37.

Yamamoto, T., Yoh, K., Kobayashi, A., Ishii, Y., Kure, S., Koyama, A., Sakamoto, T. Sekizawa, K., Motohashi, H., Yamamoto, M., 2004. Identification of polymorphisms in the promoter region of the human NRF2 gene. Biochem. Biophys. Res. Commun. 321, 72-79.

Yates, F.E., 1994. Order and complexity in dynamical systems: homeodynamics as a generalized mechanics for biology. Math. Comput. Model. 19, 49-74.

Yen, W.L., Klionsky, D.J., 2008. How to live long and prosper: autophagy, mitochondria, and aging. Physiology 23, 248-262.

Yeung, F., Hoberg, J.E., Ramsey, C.S., Keller, M.D., Jones, D.R., Frye, R.A., Mayo, M.W., 2004. Modulation of NF-KB-dependent transcription and cell survival by the SIRT1 deacetylase. EMBO J. 23, 2369-2380.

Yoshida, H., 2007. ER stress and diseases. FEBS J. 274, 630-658.

Zhao, Q., Wang, J., Levichkin, I.V., Stasinopoulos, S., Ryan, M.T., Hoogenraad, N.J., 2002. A mitochondrial specific stress response in mammalian cells. EMBO J. 21, 4411-4419.

Zhao, Y., Gong, S., Shunmei, E., Zou, J., 2009. Induction of macroautophagy by heat. Mol. Biol. Rep. 36, 2323-2327. 